\title{
Theory of a simple and efficient method for the axial optical vortex beam synthesis from a quasi-plane wave
}

\author{
A.N. Khoroshun \\ Volodymyr Dahl East-Ukrainian National University \\ 20a, Molodizhny kvartal, 91034 Luhans 'k, Ukraine \\ E-mail:an_khor@mail.ru
}

\begin{abstract}
The diffraction light field is studied by the numerical calculations of Kirchhoff-Fresnel integral and Fourier analysis. Based on combination of these methods, the condition of an axial optical vortex generation in a quasi-plane wave by the special case of a phase addition in the cross-section of a beam is founded. Amplitude and phase structures of the beam synthesized like a singular one are analyzed using computer simulation. The coefficient of conversion of an axial optical vortex beam from the quasiplane wave is defined by the ratio of intensities and reaches $65 \%$ in the far field diffraction zone. Offered are a simple original technique for the experimental realization of the necessary phase addition to the wave for the axial optical vortex beam synthesis and a light "lock" for trapping the absorbing microparticles by using two inclined mirrors.
\end{abstract}

Keywords: optical vortex, dislocation of a wave front, singular beam, diffraction, synthesis of a vortex beam, mirror.

Manuscript received 17.02.09; accepted for publication 14.05.09; published online 15.05.09.

\section{Introduction}

The dislocations of the wave front are the main object for researches in the scalar light field singular optics - a branch of modern physical optics [1-4]. Continuous dislocation lines in the light field are characterized by zero amplitude, undetermined phase and energy circulation around them. The arrangement of the line in a light beam across the axis of its propagation is called edge dislocation and along this axis - a screw dislocation or an optical vortex (OV). A simple case of $\mathrm{OV}$ beam is the Laguerre-Gaussian mode. A very stable structure of an $\mathrm{OV}$ to the intensity and phase fluctuations allow to use them as the information transmitter through the turbulence atmosphere, high sensitive detectors of thermodynamic parameter changes and sorting devices for the sub-wavelength dimension objects. Unique features of the singular beam as the "hollow" intensity distribution and orbital angular momentum are valuable in many areas for trapping and manipulation with absorbing microparticles without damage (Ch. 3 in [3]).

Dislocations of the wave front appear at the beam periphery after diffraction of the incident light on defects and edges of optical elements. The axial OV can be synthesized by means of special techniques and devices. The generation of a singular beam as the Laguerre-
Gaussian "doughnut" mode of a resonator [5] needs high output power of the laser. A disadvantage of computersynthesized holograms [6] is low efficiency of OV in the nonzero diffraction orders. A spiral phase mask [7] and wedge $[8,9]$ with the height about one wavelength are complicated in the laboratory manufacture. So, the need in the simple and efficient method of singular beam synthesis still remains.

The aim of this paper is to find theoretically the conditions of nucleation for the high-efficient axial OV from the quasi-plane wave due to further development of the modified diffusion theory of diffraction. The important question is the confirmation of the necessary influence on the quasi-plane wave by means of the found conditions which is based on the computer simulation results of the spatial beam evolution. The suggestion of a simple technique for their experimental realization is also of significant interest.

\section{Theory}

The linearly polarized light beam propagates along Zaxis with the strength of the electric field $\vec{E}(x, y, z, t)=\vec{e} A(x, y, z) \exp (i(\omega t-k z))$, where $\vec{e}$ is the unit vector in the direction of light polarization, $\omega$ is the 
light frequency and $k$ is the wave number. In the paraxial approximation, the complex amplitude

$A(x, y, z)=u(x, y, z)+i v(x, y, z)$,

where $u(x, y, z)$ and $v(x, y, z)$ are the real and imaginary parts of an amplitude, satisfies the Leontovich parabolic equation

$$
\frac{\partial A}{\partial z}=-\frac{1}{2 i k}\left[\frac{\partial^{2} A}{\partial x^{2}}+\frac{\partial^{2} A}{\partial y^{2}}\right]
$$

The equation (2) describes a stationary problem of beam evolution in the space XYZ. In the diffusion theory of diffraction [10], the relation $z=c t$ connects the distance of beam propagation $z$ with time $t$ and gives diffusion equation after substituting it in (2). The diffracted field is described by the widening of the amplitude $|A|$ on the front of a wave during its propagation. Let's briefly remind the theoretical background of the modified diffusion model of diffraction, developed in the papers $[8,11,12]$. It is based on introduction of a formal time $\tau \equiv z$ for the convenience of beam space evolution studying due to coincidence of time and distance. As a result of substitution of the expression (1) into equation (2) in the form of $z=\tau$, obtained is the system of equations for the field components $u(x, y, z)$ and $v(x, y, z)$, which describe the diffusion of real and imaginary parts of amplitude on the front of a wave at the plane $X Y$ in time $\tau$

$$
\left\{\begin{array}{l}
\frac{\partial u}{\partial \tau}=\frac{1}{4}\left(\frac{\partial^{2} v}{\partial \tilde{x}^{2}}+\frac{\partial^{2} v}{\partial \tilde{y}^{2}}\right), \\
\frac{\partial v}{\partial \tau}=-\frac{1}{4}\left(\frac{\partial^{2} u}{\partial \tilde{x}^{2}}+\frac{\partial^{2} u}{\partial \tilde{y}^{2}}\right)
\end{array}\right.
$$

For the sake of convenience in the paper, all the transverse coordinates $x, y$ are normalized on $r_{0}$, where $r_{0}$ is the beam waist radius at $\tau=0$, and the longitudinal coordinate $z=\tau$ in the Rayleigh range $z_{R}=k r_{0}^{2} / 2$.

On the one hand, the real $u\left(x_{1}, y_{1}, \tau\right)$ and the imaginary $v\left(x_{1}, y_{1}, \tau\right)$ parts of complex amplitude at the time $\tau$ can be defined by the Kirchhoff integrals

$$
\begin{aligned}
& u\left(x_{1}, y_{1}, \tau\right)=\operatorname{Re}\left(\frac{k}{2 \pi i} \iint A_{0}(\tilde{x}, \tilde{y}) \frac{\exp \left(i k r_{0} r\right)}{r_{0} r} d \tilde{x} d \tilde{y}\right), \\
& v\left(x_{1}, y_{1}, \tau\right)=\operatorname{Im}\left(\frac{k}{2 \pi i} \iint A_{0}(\tilde{x}, \tilde{y}) \frac{\exp \left(i k r_{0} r\right)}{r_{0} r} d \tilde{x} d \tilde{y}\right),
\end{aligned}
$$

where $A_{0}(\tilde{x}, \tilde{y})$ is the amplitude distribution of the field at the source plane, $r$ is a module of the radius vector, $r=\sqrt{\left(\tilde{x}-x_{1}\right)^{2}+\left(\tilde{y}-y_{1}\right)^{2}+\left(\tau z_{R} / r_{0}\right)^{2}}, \quad \tilde{x}, \tilde{y}$ are the coordinates of the point in the source plane $\tau=0$ and $x_{1}, y_{1}$ are the coordinates of the point in the diffraction pattern plane $\tau>0$.
On the other hand, $u\left(x_{1}, y_{1}, \tau\right)$ and $v\left(x_{1}, y_{1}, \tau\right)$ functions can be represented by the Fourier integrals

$$
\begin{aligned}
& u\left(x_{1}, y_{1}, \tau\right)=\iint u_{0}\left(\hat{k}_{\perp x}, \hat{k}_{\perp y}\right) \\
& \times \exp \left(-i\left(\hat{\omega}_{\perp} \tau z_{R}-\hat{k}_{\perp x} r_{0} \tilde{x}-\hat{k}_{\perp y} r_{0} \tilde{y}\right)\right) d \hat{k}_{\perp x} d \hat{k}_{\perp y}, \\
& v\left(x_{1}, y_{1}, \tau\right)=\iint v_{0}\left(\hat{k}_{\perp x}, \hat{k}_{\perp y}\right) \times \\
& \times \exp \left(-i\left(\hat{\omega}_{\perp} \tau z_{R}-\hat{k}_{\perp x} r_{0} \tilde{x}-\hat{k}_{\perp y} r_{0} \tilde{y}\right)\right) d \hat{k}_{\perp x} d \hat{k}_{\perp y},
\end{aligned}
$$

where subintegral functions are the plane waves propagating along $\mathrm{XY}$ direction, $\omega_{\perp}$ is the frequency of the harmonic spreading in the direction of the transversal wave number $\hat{k}_{\perp}=\sqrt{\hat{k}_{\perp x}^{2}+\hat{k}_{\perp y}^{2}}$ defining the distance $0 \leq L \leq \infty$ in the plane $\mathrm{XY}$, on which the phase is changed by $2 \pi, \hat{k}_{\perp}=2 \pi / L$. It should be emphasized that there is no connection between the transversal wave number $\hat{k}_{\perp}$ and light wave number $k$ as in Eq. (2).

The initial light field with symmetric amplitude and phase distributions at the time $\tau=0$ is considered. For simplicity, it can be shown for a one-dimensional case of the beam distribution, for example along Y-axis. The nonperiodic functions $u(\tilde{y}, \tau)$ and $v(\tilde{y}, \tau)$ can be represented by the Fourier integral at the time $\tau=0$

$$
\begin{aligned}
& \left.u(\tilde{y}, 0)=\frac{1}{2 \pi} \int_{-\infty}^{\infty} u\left(\hat{k}_{\perp}, 0\right) \exp \left(-i \hat{k}_{\perp} r_{0} \tilde{y}\right)\right) d \hat{k}_{\perp} \text { and } \\
& v(\tilde{y}, 0)=\frac{1}{2 \pi} \int_{-\infty}^{\infty} v\left(\hat{k}_{\perp}, 0\right) \exp \left(-i \hat{k}_{\perp} r_{0} \tilde{y}\right) d \hat{k}_{\perp} .
\end{aligned}
$$

Then, the Fourier transform of the functions are

$$
\begin{aligned}
& u\left(k_{\perp}, 0\right)=\int_{-l}^{l} u(\tilde{y}, 0) \exp \left(i \hat{k}_{\perp} r_{0} \tilde{y}\right) d \tilde{y} \text { and } \\
& v\left(k_{\perp}, 0\right)=\int_{-l}^{l} v(\tilde{y}, 0) \exp \left(i \hat{k}_{\perp} r_{0} \tilde{y}\right) d \tilde{y},
\end{aligned}
$$

where $2 l$ is the distance of the amplitude functions existing along $\mathrm{Y}$-axis.

So, the resulting features of a diffraction field can be predicted by verification of transversal wave numbers $k_{\perp}$. The nonperiodic functions $u(\tilde{y}, 0)$ and $v(\tilde{y}, 0)$ are described by continuous amplitude spectra $A\left(\hat{k}_{\perp}\right)$ defined by the formulas

$$
A_{u}\left(\hat{k}_{\perp}\right)=2\left|u\left(\hat{k}_{\perp}\right)\right|, \quad A_{v}\left(\hat{k}_{\perp}\right)=2\left|v\left(\hat{k}_{\perp}\right)\right| .
$$

The relative amplitude spectra $A\left(\hat{k}_{\perp}\right) / A_{\max }\left(\hat{k}_{\perp}\right)$ of the real and imaginary amplitude functions show the contribution of every harmonic $L=2 \pi / \hat{k}_{\perp}$ to the initial 
total field. The transversal phase dependence in the nondimensional variables on every harmonic attains the form

$\Phi_{\perp}(\tilde{y}, 0)=\hat{k}_{\perp} r_{0} \tilde{y}$,

where the value of transversal wave number changes as $0<\hat{k}_{\perp}<\infty$.

The spectral width $\Delta \hat{k}_{\perp}$ is the interval of wave numbers, over which the spectral amplitude differs significantly from zero [13]. In what follows, we assume that the spectral width defines the basic harmonic $L_{0}=2 \pi / \Delta \hat{k}_{\perp}$ that reveals the primary beam phase structure

$$
\Phi_{0}(\tilde{y}, 0)=\Delta \hat{k}_{\perp} r_{0} \tilde{y}
$$

\section{The synthesis of a singular beam from a quasi-plane wave}

The main aim of the theoretical investigation based on the foregoing model is to search out the conditions for radical reconstruction of the initially smooth wave front into the helical one, which is typical to an axial optical vortex. As a starting field, a quasi-plane wave is examined, which is often used in the theoretical and the experimental wave process investigations as well as in the holography researches and its applications.

The examples of waves containing optical vortices are the Laguerre-Gaussian modes $L G_{0}{ }^{m}$ :

$$
\begin{aligned}
& L G_{0}^{m}(\rho, \varphi, \tau)=\frac{A_{0}}{\sqrt{1+\tau^{2}}}\left(\frac{\rho}{\sqrt{1+\tau^{2}}}\right)^{|m|} \exp \left(-\frac{\rho^{2}}{1+\tau^{2}}\right) \times \\
& \times \exp i\left[\frac{\rho^{2} \tau}{\sqrt{1+\tau^{2}}}-m \varphi-(|m|+1) \operatorname{arctg} \tau+k \tau \cdot z_{R}\right],
\end{aligned}
$$

where $A_{0}$ is the amplitude parameter, $\rho=\sqrt{\tilde{x}^{2}+\tilde{y}^{2}}, \varphi$, $\tau$ are cylindrical nondimensional coordinates, $m$ is the topological charge of an $\mathrm{OV}$ and $(|m|+1) \operatorname{arctg} \tau$ is the Gouy phase shift. The wave front of an OV has a helicoid's form, the axis of which coincides with the axis of beam propagation. The sign of a dislocation charge points the direction of energy circulation in the beam, and its amount defines the changing value of the phase $\Phi$ represented as a multiplier before the complex unity in (11), around the dislocation line in a view $m=\frac{\oint d \Phi}{2 \pi}$. The field distribution of a singular beam with the positive single charge at the time $\tau=3$ is shown in Fig. 1. All the calculations are made for $\mathrm{He}-\mathrm{Ne}$ laser $(\lambda=0.6328 \mu \mathrm{m})$ with the beam waist $r_{0}=0.025 \mathrm{~mm}$.

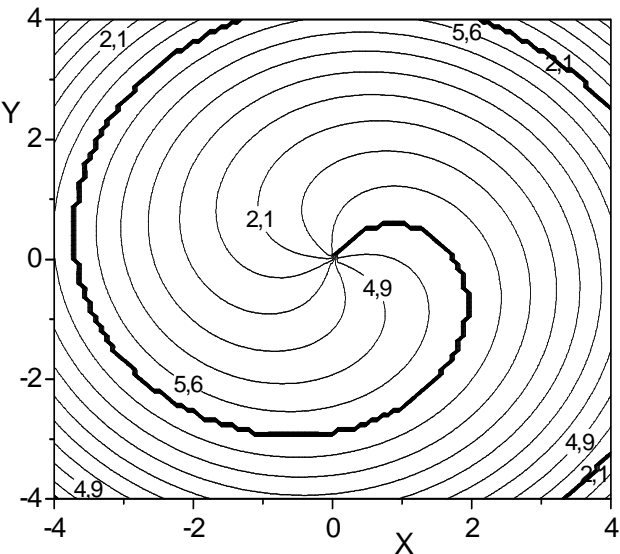

(a)

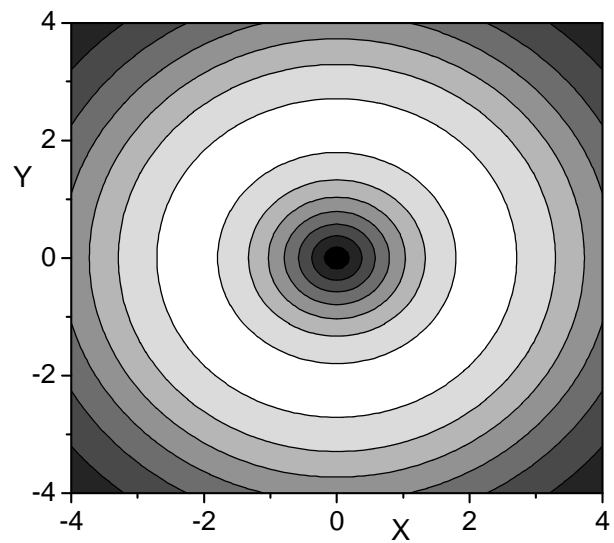

(b)

Fig. 1. Phase map $(a)$ and amplitude distribution $(b)$ of the singular beam $\left(L G_{0}{ }^{1}\right.$ mode) with the topological charge $m=+1$ that can be seen due to phase growing counterclockwise by the value of $2 \pi$.

The plane monochromatic wave with a light frequency $\omega$ propagating along $Z$-axis can be written as

$E(z, t)=A(x, y, z) \cos \left(\omega t-k z+\varphi_{0}\right)$,

where the constant value of amplitude $A(x, y, z)$ is supposed to be equal to unity, $k$ is a wave number and $\varphi_{0}$ is an initial phase of the wave. Let's, for simplicity, consider some one-dimensional case (the choice of $\tilde{x}$ or $\tilde{y}$-component is of no significance) of the field representation. It's assumed the equality of the wave amplitude to its real part $A(\widetilde{x}, 0)=u(\widetilde{x}, 0)$ at the time $\tau=0$. Then, the imaginary part of the wave amplitude $v(\tilde{x}, 0)$ is equal to zero due to our choice and the relation (1) for the complex amplitude. The first term of the argument of harmonic function in (12) gives fast oscillation, which doesn't influence on the form of the wave front. So, the constant-phase surfaces represent the infinite planes that continuously fill space along all the paths of wave propagation starting from the phase value $\varphi_{0}$ at the time $\tau=0$. Only the wave limited in space with the characteristic radius $r_{0}$ is experimentally realized. 
Such plane wave is called the quasi-plane wave, which is considered in this paper. A sharp amplitude shock in the region near $r_{0}$ is typical for the diffraction problem of a plane wave by a circular aperture. Propagation of a plane wave can be described by the gradually amplitude decreasing within the region near $r_{0}$, which is taken into account due to providing very narrow Gaussian profile (Fig. 2a). The amplitude distribution of the quasi-plane wave with the radius $r_{0}=1$ can be represented in the form:

$$
u(\tilde{x}, 0)=\left\{\begin{array}{cc}
1, & |\tilde{x}| \leq 1 \\
\exp \left(-(\tilde{x}-1)^{d}\right), & \tilde{x}>1 \\
\exp \left(-(\tilde{x}+1)^{d}\right), & \tilde{x}<-1
\end{array},\right.
$$

where $d=20$ is the power value, which provides a gradually amplitude decrease within the region near $r_{0}=1$. The choice of the power value $d$ in (13) is arbitrary.

The relative amplitude spectrum $A\left(\hat{k}_{\perp}\right) / A_{\max }\left(\hat{k}_{\perp}\right)$ of the function $u(\widetilde{x}, 0)$ similar to the spectrum of single rectangular pulse is numerically calculated using the expressions (10), (11) and represented in Fig. 2b. The spectral width $\Delta \hat{k}_{\perp}$ is the

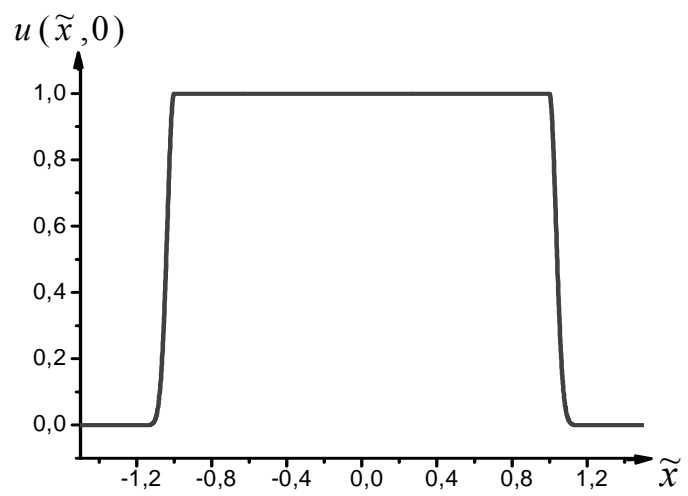

(a)

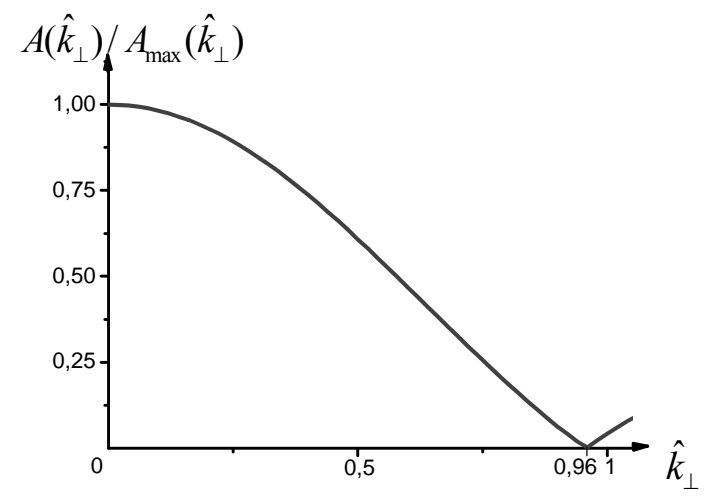

(b)

Fig. 2. Amplitude distribution $u(\tilde{x}, 0)$ of a quasi-plane wave $(a)$ and its spectrum $A\left(\hat{k}_{\perp}\right)$ depends on the transversal wave number $\hat{k}_{\perp}(b)$ at the time $\tau=0$. interval of the transversal wave numbers from zero up to the wave number at which the amplitude becomes equal to zero for the first time. So, the value of spectral width $\Delta \hat{k}_{\perp} \approx 0.96$ is measured in Fig. 2b. Then according to the reasoning (13), the main transversal phase dependence takes the form

$$
\Phi_{0}(\tilde{x}, \tilde{y}, 0)=\Delta \hat{k}_{\perp} r_{0} \tilde{x}=0.96 r_{0} \tilde{x}
$$

We used two statements when developing the method for synthesis of the axial OV from the quasiplane wave. 1) The appearance of an $\mathrm{OV}$ in the light field is caused by the destructive interference formed by many wave microsources. 2) Around the dislocation line in the cross-section of a beam, the phase changes by $2 \pi$. In the simplest case of two monochromatic waves linearly polarized in the same direction, destructive interference occurs when their amplitudes are equal and phases differ by $\pi$. So, let's divide the quasi-plane wave cross-section into two areas $(\tilde{y} \leq 0, \tilde{x})$ and $(\tilde{y}>0, \tilde{x})$ with the identical amplitudes as two sources for interference. Synthesis of the axial OV requires that the phase has to grow by $2 \pi$ along $\mathrm{X}$-axis and change by $\pi$ only in the center of a beam. The value of the distance $L$ along $\mathrm{X}$-axis, on which the phase grows by $2 \pi$, is calculated from the formula (10). So, the conditions of synthesis for the singular beam with the topological charge $m=+1$ from the initial quasi-plane wave can be written in the form

$$
\Phi(\tilde{x}, \tilde{y}, 0)= \begin{cases}\varphi_{0}-\Phi_{0}(\tilde{x}, \tilde{y}, 0)-\pi / 2, & \tilde{y}>0, \\ \varphi_{0}+\Phi_{0}(\tilde{x}, \tilde{y}, 0)+\pi / 2, & \tilde{y} \leq 0 .\end{cases}
$$

As a result of phase varying on the way offered in (15), the quasi-plane wave transforms into a beam with an axial optical vortex with the topological charge $m=+1$ that can be determined from the phase growing from 0 to $2 \pi$ counterclockwise (Fig. $3 \mathrm{a}$ ). The simulations show that the best result of synthesis is achieved when the spectral width value $\Delta \hat{k}_{\perp}=1 \pm 0.04$. The founded value of $\Delta \hat{k}_{\perp}=0.96$ belongs to the confidence interval. Such insignificant distinction of results can be explained by the initial quasi-plane wave approach. On the one hand, a gradually amplitude decrease makes wave widening with time in computer simulations (Fig. 4b, c), which is not typical for an ordinary experimentally obtained plane wave. On the other hand, sharp amplitude shock in the region $r_{0}=1$ gives right wave representation at the time $\tau=0$. As the main result of the theory is found for the initial field, the spectral width value can be considered as equal to unity, $\Delta \hat{k}_{\perp}=1$.

Two diffraction amplitude maxima on the $\mathrm{X}$-axis (Fig. 3b), which are matched by dash line, shift clockwise with time (Fig. 4b, c). The amplitude asymmetry of the obtained beam at the time $\tau=1$ decreases with time, and field characteristics weakly change after the time $\tau=3$ (Fig. 4b, c). Amplitude and 
phase distributions are enough similar to the singular beam with the topological charge $m=+1$ already beginning from the time $\tau=3$ (Fig. 3b, c). The ellipticity amplitude $\gamma$ is caused by two asymmetric beams propagating in the opposite directions. This value can be measured by the ratio of the small to big ellipse semiaxes $\gamma=b / a \approx 0.65$, which are drawn through the common amplitude contour line and the beam center (Fig. 4b). The optical vortex core ellipticity can be useful for trapping the rod-shaped objects like bacteria and polymeric microparticles.

The efficiency coefficient $\eta$ of the axial OV beam synthesis from a quasi-plane wave is defined by the ratio

$\eta=\frac{I_{\min }}{I_{\max }} \cdot 100 \%$,

where $I_{\min }$ is the minimal intensity in the area of maximum intensity $I_{\max }$ of the obtained $\mathrm{OV}$ beam at the same time $\tau$ (Fig. $3 \mathrm{~b}$ ). The value of the conversion coefficient is equal to $\eta=(65.2 \pm 0.1) \%$ at the time $\tau=3$.

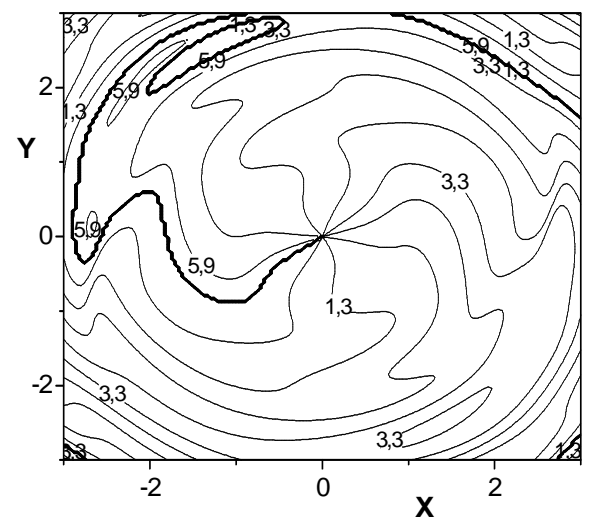

(a)

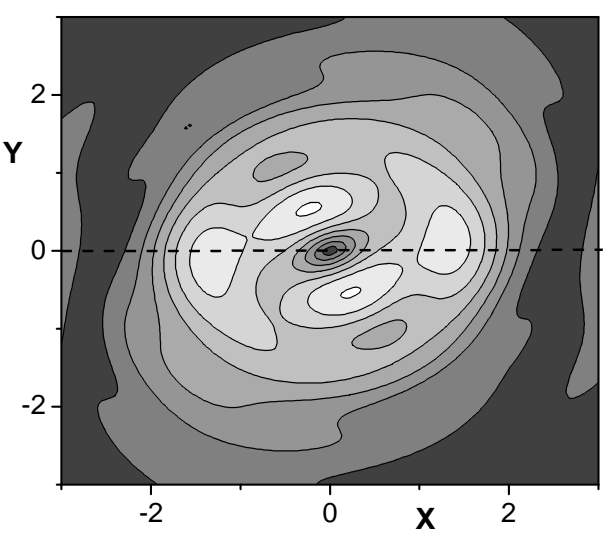

(b)

Fig. 3. Phase $(a)$ and amplitude $(b)$ distributions in the transverse section of an axial OV beam synthesized after the phase addition to the quasi-plane wave at the time $\tau=1$ and the spectral width value $\Delta \hat{k}_{\perp}=1$, dashed line in $(b)$ shows the boundary between decreasing and increasing phase additions to the initial wave.

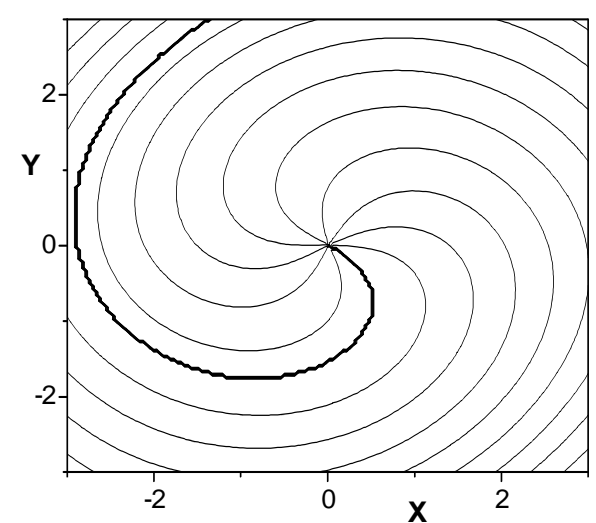

(a)

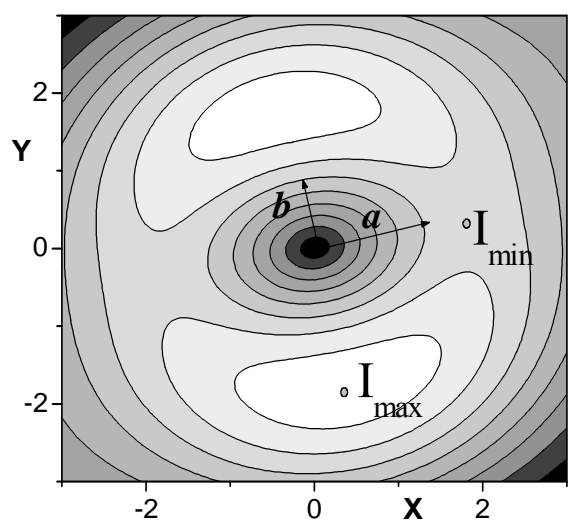

(b)

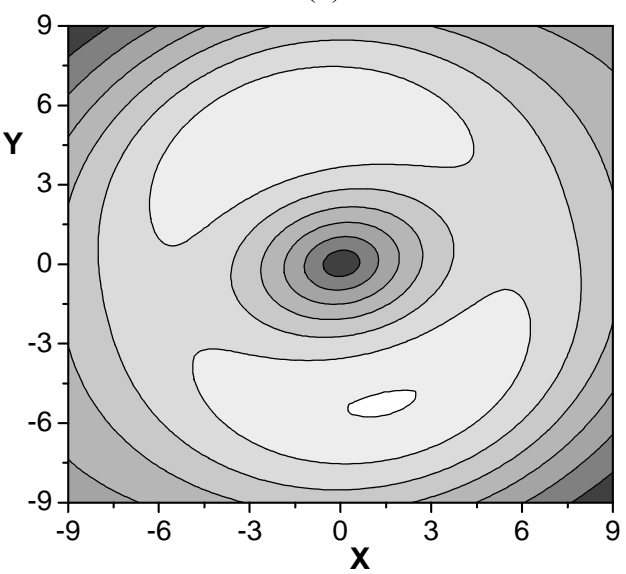

(c)

Fig. 4. Results of calculations of phase (a) at the time $\tau=3$ and amplitude distributions in the transverse section of an axial optical vortex beam synthesized from a quasi-plane wave due to the phase addition with the spectral width value $\Delta \hat{k}_{\perp}=1$ at the time $\tau=3(b)$ and $\tau=10(c)$.

In the case of the spectral width value $\Delta \hat{k}_{\perp}$ increasing with the constant phase difference by $\pi$ in the center, the topological wave front features and the intensity pattern of the beam are changed. The edge dislocation seen in the interferogram by the fringes 
shifted by $\pi$ at $\mathrm{Y}$-axis at the spectral width $\Delta \hat{k}_{\perp}=0.02$ (Fig. 5a) transforms into the screw dislocation detected by the fringe "fork" (Fig. 5b). The low conversion coefficient $\eta, \quad$ namely, $\eta\left(\Delta \hat{k}_{\perp}=0.1\right) \approx 1 \% \quad$ and $\eta\left(\Delta \hat{k}_{\perp}=0.25\right) \approx 6 \%$, denotes noticeable amplitude asymmetry under these parameters of $\Delta \hat{k}_{\perp}$. Amplitude distribution of the obtained axial OV has the form of two stable "hills", which rotate clockwise from the X-axis to $\mathrm{Y}$-axis with increasing $\Delta \hat{k}_{\perp}$ (Figs. 6a, 6b, 4b). The area of the minimal intensity from the beam periphery into the axial OV beam centre (Fig. 6a) is the way of the absorbing microparticle movement. These features can be used as a light lock of the particle: trapping the object along $\mathrm{Y}$-axis at $\Delta \hat{k}_{\perp}=0.25$ and holding the particle by the growing spectral width up to $\Delta \hat{k}_{\perp}=1$ (Fig. $4 b$ ).

The synthesis of an axial OV beam from a quasiplane wave and a light lock can be realized experimentally by the technique of two inclined mirrors (Fig. 6). The primary beam propagates along $\mathrm{Z}$-axis and reflects from the mirror $M_{1}$ is turned by the angle $-\alpha / 2$ to the plane $\mathrm{XY}$ in the area $\tilde{x}<0$ and the mirror $M_{2}$ turned by the angle $\alpha / 2$ to the plane $X Y$ in the area $\tilde{x} \geq 0$. The necessary phase difference $\Delta \Phi_{0}=\Delta \hat{k}_{\perp} \tilde{x}$ is acquired for the angle $\alpha=\Delta \hat{k}_{\perp} r_{0} / 2 z_{R}$, if taking into account the double-ray path in the dimensionless variables. The axial OV beam synthesis with the amplitude distribution like a singular beam with the topological charge $m=+1$ from a quasi-plane wave should appear under the condition $\alpha=\lambda / 2 \pi r_{0}$. By the changing the angle $\alpha$, the necessary amplitude rotation or a light lock of a microparticle are reached.

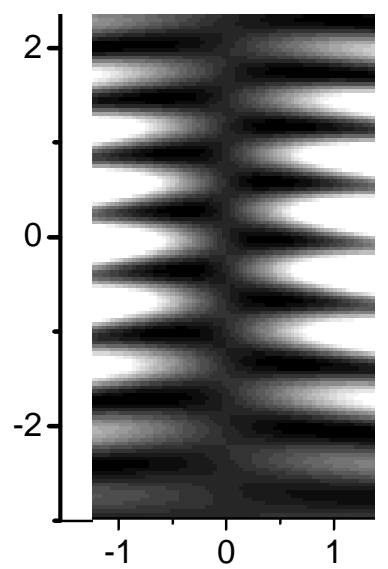

(a)

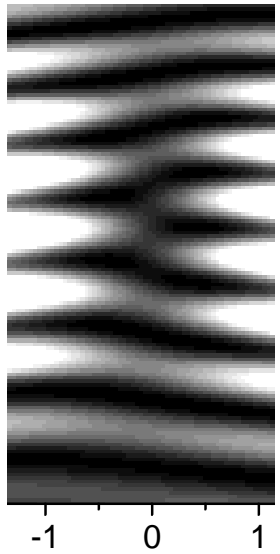

(b)
Fig. 5. Patterns of obtained beam interference with a plane reference wave for the spectral widths $\Delta \hat{k}_{\perp}=0.02(a)$ and $\Delta \hat{k}_{\perp}=1(b)$ at the time $\tau=3$.

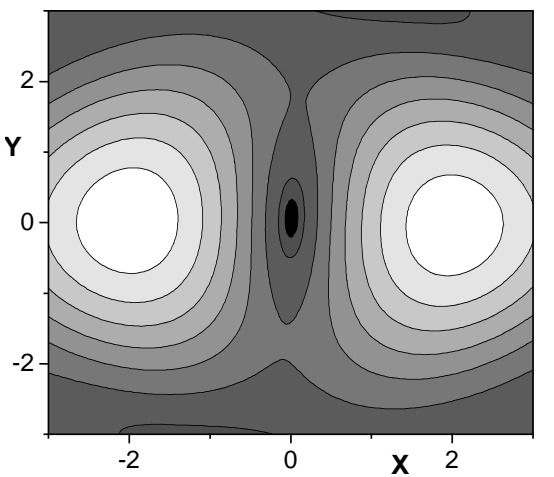

(a)

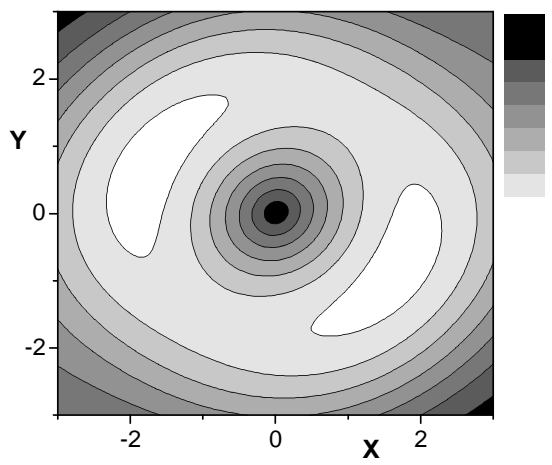

(b)

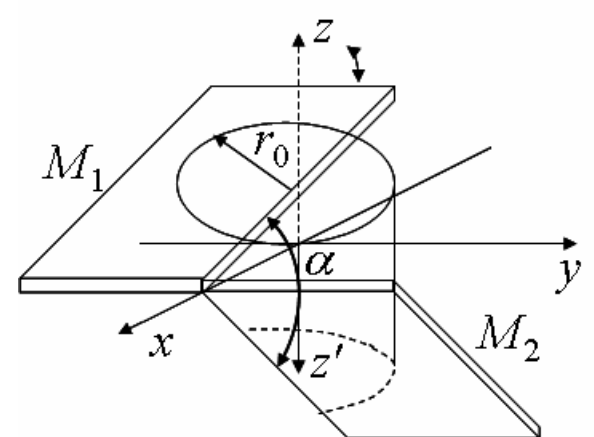

(c)

Fig. 6. Amplitude distributions in the transverse section of an axial OV beam synthesized with the spectral width $\Delta \hat{k}_{\perp}=0.25(a)$ and $\Delta \hat{k}_{\perp}=0.8(b)$ at the time $\tau=3$. (c) Possible arrangement of experimental mirrors for the synthesis of an axial optical vortex from a quasi-plane wave and a light lock of a microparticle in the reflected beam that propagates along $\mathrm{Z}$-axis.

\section{Conclusions}

In this paper, the method of an axial optical vortex generation in a quasi-plane wave by the special case of a phase addition in the cross-section of a beam has been founded by analyzing the amplitude spectral composition using Fourier transformation. The initial field amplitude is represented as the sum of the plane waves propagating with the different wave numbers $\hat{k}_{\perp}$ on the plane $X Y$ at the time $\tau=0$. The width of 
amplitude spectrum $\Delta \hat{k}_{\perp}=1$, by the author opinion, defines the basic harmonic in the initial field distribution and the necessary phase changing to the plane wave front for its transformation into the helical one.

The evolution of a quasi-plane wave after phase addition has been numerically calculated by the Kirchhoff-Fresnel integral in the far field diffraction zone. At small values of amplitude spectrum width, for example $\Delta \hat{k}_{\perp}=0.02$, the edge dislocation appears due to an almost constant value of the phase shock along the $\mathrm{X}$-axis. The dislocation transforms into the screw dislocation with growth of $\Delta \hat{k}_{\perp}$. The best synthesized beam intensity and phase parameters in comparison with the singular beam of a single topological charge were obtained for $\Delta \hat{k}_{\perp}=1 \pm 0.04$. Asymmetry of the radial amplitude distribution of the obtained axial OV beam is defined by the conversion coefficient that continuously changes depending on the width of the amplitude spectrum from $1 \%$ at $\Delta \hat{k}_{\perp}=0.1$ up to $65.2 \%$ at $\Delta \hat{k}_{\perp}=1$.

The original method of an axial OV beam synthesis from a quasi-plane wave has been suggested. The necessary phase addition in the cross-section of a beam is produced using the technique of two inclined mirrors. A light lock for trapping the absorbing microparticle has been offered. It is based on the dependence of the angle between inclined mirrors or the value of spectral width $\Delta \hat{k}_{\perp}$ on the obtained amplitude pattern of the synthesized beam.

\section{Acknowledgements}

The author is grateful to Prof. V. Gorshkov and Prof. $M$. Vasnetsov for the previous scientific advising and the critical and stimulating discussions concerning to the methodology of making research as well as to Prof. M. Soskin for the introduction into the field of Singular Optics.

\section{References}

1. 1. J.F. Nye, M.V. Berry // Proc. R. Soc. London A 336, p. 190 (1974).

2. M.S. Soskin, M.V. Vasnetsov // Progr. Opt. 42 (2001) 276.

3. M. Vasnetsov, K. Staliunas, Horizons in World Physics. Nova Science Publ. 228, p. 313 (1999).

4. A.Ya. Bekshaev, M.V. Vasnetsov, M.S. Soskin // Ukr. J. Phys.: Rev. 2, p. 73-113 (2005).

5. J.M. Vaughan, D.V. Willets // Opt. Communs. 30, p. 263-267 (1979).

6. V.Yu Bazhenov, M.V. Vasnetsov, M.S. Soskin // JETP Lett. 52, p. 1037-1039 (1990).

7. M.W. Beijersbergen, R.P.S. Coerwinkel, M. Kristensen, J.P. Woerdman // Opt. Communs. 112, p. 321-327 (1994).

8. V.N. Gorshkov, A.N. Khoroshun, M.S. Soskin // Proc. SPIE 4705, p. 65-74 (2002).

9. Ya. Izdebskaya, V. Shvedov, D. Kurabtzev, A. Alexeyev, A. Volyar // Proc. SPIE 4607, p. 7882 (2002).

10. V.N. Gorshkov, A.N. Khoroshun, M.S. Soskin // Ukr. J. Phys. 51, No. 2, p. 134-141 (2006).

11. V.N. Gorshkov, A.N. Khoroshun, M.S. Soskin // Ukr. J. Phys. 50, No. 4, p. 351-360 (2005).

12. M.A. Leontovich and V.A. Fock // Zhurnal Eksperiment. Teor. Fiziki 16, No. 7, p. 557-573 (1946) (in Russian).

13. A.N. Matveev, [translated from Russian by Ram Wadhma] Optics. Mir Publishers, Moscow, 1988, p. 446. 